\title{
Simulation of the Single Point Incremental Forming of Polyamide and Polyethylene Sheets
}

\author{
Nicolae Alexandru Roșca ${ }^{1, *}$, and Mihaela Oleksik ${ }^{1}$ \\ ${ }^{1}$ Faculty of Engineering, Department of Industrial Engineering and Management, „Lucian Blaga” \\ University, Sibiu, Romania
}

\begin{abstract}
The present paper aims the theoretical study, using the finite element method, on the single point incremental forming process of two polymeric materials: polyamide 6.6 and high-density polyethylene. The experimental researches used for the determination of the true stress - true strain curves for two materials are presented, which are necessary for their introduction into the simulation. The explicit analysis is carried out with the Ls-Dyna program and the results of the analysis were focused on the major strain, minor strain, thickness reduction, forces on the process and total energy consumed in the process.
\end{abstract}

\section{Introduction}

The field of application of cold plastic deformation processes is particularly developed and continues to develop, covering a wide range in the machinery and apparatus industry, electrotechnics and electronics, fine mechanical, consumer goods, chemical industry machinery and food, etc.

Among the cold plastic forming processes, the plate deformation processes have become increasingly widespread in recent years. The deep-drawing and stretching processes offer great possibilities for making three-dimensional parts with high stiffness properties. In small or unique series production, however, the very high specific costs of tools and machines are felt to be negative.

The geometrical flexibility of the forming processes is currently the highest abstraction step in the choice of semi-finished products, tools and machines. The desired contour of the piece no longer exists as a template, materialized on tool. The final geometry of the piece is stored as abstract information on a magnetic data carrier. The information is transposed as a set of processing parameters on a universal machine tool that performs a simple, universal tooling whose shape and dimensions are not specific to the piece. Spatial adjustments are made on separate motion axes. Thus, by means of "geometrically flexible" systems, a piece is not performed in a single pass (in a single step), but through a succession of time in several individual stages.

In the process of incremental forming of the metal sheets, deformation is accomplished by a die that comes in partial contact with the surface of the blank. To achieve the shape of the piece, one of the active elements (generally the die) will have an axial, continuous or

${ }^{*}$ Corresponding author: nicolae.rosca@ulbsibiu.ro 
stepwise (incremental) movement in the vertical direction while the other (active plate) will perform a horizontal planar motion. The flexibility of the process is great because with the same die and the same active plate, depending on the movements imposed on the active elements, using the same machine tool, a multitude of cave shapes can be obtained [1].

Most scientific work in this field, of single point incremental forming, are works that analyze experimentally or theoretically the main deformations occurring in the process (major strain, minor strain or thickness reduction) and the influence of the factors of geometric parameters (the die diameter, the vertical step, the type of trajectory) on these parameters, to metallic materials and their alloys. [2-3] Since the SPIF process has as its main disadvantage the low precision, several recent works have analyzed various possibilities to improve it by optimizing the die trajectories [4-5].

Due to the flexibility of the process and the increase of the spreading area, it is desirable to evaluate the behavior of other types of materials (non-metallic materials) in this process. Therefore, the first experimental researches were carried out to determine the forces in the process and the influence of the temperature on the SPIF deformation of the PVC [6].

The latest research on polymeric materials is related to the use of cranial implants from polymeric biocompatible materials made by single point incremental forming [7].

Even though there have recently been researches on SPIF processing of polymeric materials, there is not enough theoretical research related to the SPIF processing of these materials. Therefore, this paper proposes precisely such a study, an analysis using the finite element method of the SPIF behavior of the two types of materials.

\section{Machines and experimental stands used to determine material properties}

To determine the data of the material needed to simulate the incremental forming process of plastic materials, we have used the uniaxial stretch test.

The oldest method of material behavior testing is the uniaxial stretch test. The specimen is fixed at both ends and deformed at a constant speed, on a traction test machine until breaks. The applied force is measured with a force cap and the deformation by an extensometer.

The data obtained can be plotted directly in the force-displacement coordinates. In many situations, they are converted into stress-strain coordinates. In case of the uniaxial stretch test of laminated plastic materials, we have chosen to collect the data in the form of forceelongation pairs.

By coupling the Instron 5587 traction, compression and buckling machine with the optical measurement system it was no longer necessary to use an extensometer, the Aramis optical system being a high precision optical extensometer.

To determine the anisotropy of the material during the uniaxial stretching test, deformations of the specimens were measured using the Aramis optical analyzer. The optical measurement system has been positioned and calibrated so that the measuring area is large enough to allow the two chambers of the system to fully register the test process to which the specimens are subjected.

To carry out experimental research with the Aramis measuring system it is necessary to deposit in advance a network on the surface of the workpiece. If in the case of metal sheets, sheets or other materials (generally rigid), it is preferable to lay a layer of matte white paint on the surface of the workpiece to eliminate undesirable reflections and then to deposit a graphite point grid, in the case of plastics due to their white color, we eliminated the painting process. Due to the specificity of this type of test (very high deformation rates in the direction of stress) we found that the white paint exfoliated before the rupture occurred and thus appeared the impossibility of determining the deformations. Therefore, the 
preparation of the specimens consisted only in the application of a diffuse graphite dust network. With the prepared specimens, the testing process is started, and the number of images desired by the user is acquired. In the case of present research, the CCD camera acquisition rate was set to 1 image/second, as recommended by the literature. When the cracks are beginning to be seen, the recording stops. At the end of the test process, the area to be measured is determined, eliminating unnecessary areas in the measurement process, and then is set (automatically or by the user) a reference point (starting point) for the calculation of deformations. It should be noted that this point must be present in all the images acquired during the test and that the setting of this point has no influence on the accuracy of the measurement. After processing all images, the optical measurement system determines the displacements after the three axes of the coordinate system of each point on the specimen. By making additional calculations, the unknowns are determined, namely the elongations (or shortenings) along the $\mathrm{x}, \mathrm{y}$ and $\mathrm{z}$ directions as well as the specific rotation. By rotating the coordinate axes trihedral to eliminate specific rotation, the main and secondary deformations, the relative thinning (where appropriate), the shear deformations (shear angles) and the equivalent deformations Von Mises or Tresca are determined.

\section{Determination of flow curves for the two plastics}

The experimental program for determining the mechanical characteristics of PA 6.6 and PE laminates is based on the following:

- $\quad$ sets of five specimens were taken for each type of material, for example polyamide PA 6.6 and PE. The shape of the specimens was the standard for this type of test;

- the initial thickness of the specimens was $\mathrm{g}=1.4 \mathrm{~mm}$ for both materials;

- a test method was developed in the Instron test machine's own language, namely Bluehill 2. It was established at this stage: test type (traction), material data (specimen shape, specimen width, distance between machine grips), test speed, machine limits, machine acquisition rate (10 points/second), output file type (ASCII, a file format that can be downloaded from any of the statistical data processing programs), the type of output data are to be collected;

- $\quad$ the test speed was set at $10 \mathrm{~mm} / \mathrm{min}$;

- the width of the specimen was $b_{0}=20 \mathrm{~mm}$ and the free measuring distance $1_{0}=100$ $\mathrm{mm}$;

- the output data were: maximum force [N], elongation corresponding to maximum force $[\mathrm{mm}]$, tensile stress $[\mathrm{MPa}]$, tensile strain $[\mathrm{mm} / \mathrm{mm}]$, true stress $[\mathrm{Mpa}]$ and true strain $[\mathrm{mm} / \mathrm{mm}]$;

- In addition to the data specified above, the primary test data (characteristic curve in tensile-stress $[\mathrm{MPa}]$ - tensile strain $[\mathrm{mm} / \mathrm{mm}]$ ) is saved. These data are in the form of pairs of points in the coordinates listed above in the file of each ASCII analysis.

- the main deformations were also determined by the Aramis measuring system.

Figures 1 and 2 show the true stress - true strain for the two tested materials and table 1 presents the numerical results of the tests with the associated statistical processing. 

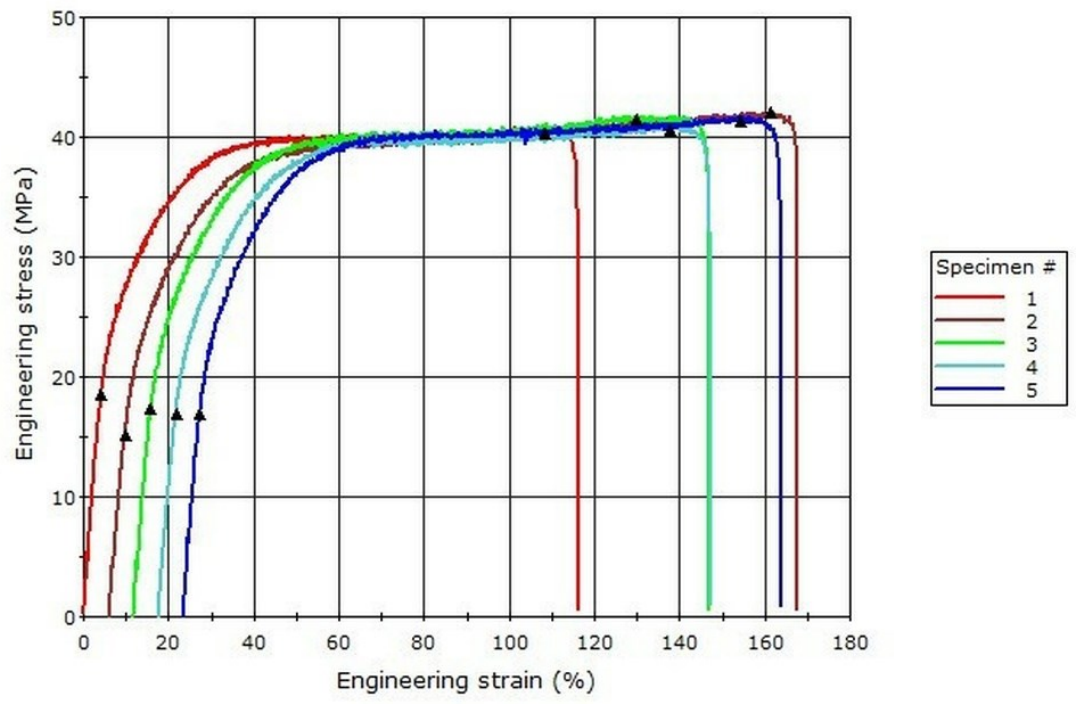

Fig. 1. The true stress - true strain graphics for polyamide
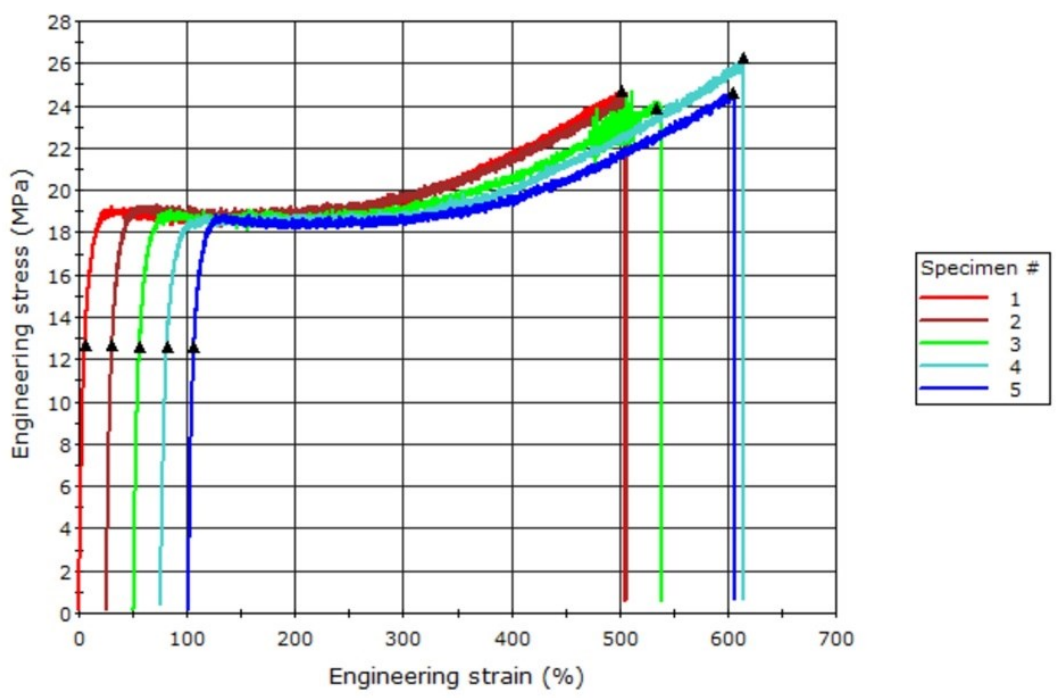

Fig. 2. The true stress - true strain graphics for polyethylene

\section{FEM simulation of the incremental forming process of plastics}

In addition to the mathematical description by analytical methods based on the theory of plasticity, the modeling of the deformation processes by numerical methods has become more and more important. Among these, the finite element method is clearly distinguished which due to the large-scale application in the field of engineering calculations, achieves a very good approximation of the results calculated by the experimentally determined ones. This success has two main causes. The first case is based on the theoretical developments of this computational method that materialize in the emergence of new programs or 
modeling facilities within the established software packages. A second cause is the development of powerful, relatively inexpensive computers that facilitate complex analyzes, specific to the finite element method.

An overview of the method of analysis used in the present paper is presented below.

In the first stage, we find the preprocessing phase of the data corresponding to the physical model of the processing process. At first, deformable and rigid bodies are defined based on the geometry of the blank and tools. Geometry is the one that is specific to the start of processing. Based on the geometry of the deformable body, it is meshed into finite elements. The set of elements thus defined is associated:

- material data, in this case the flow curve, the density of the material, the modulus of elasticity E and the coefficient of Poisson v;

- geometric data, namely geometry of tools and blank;

- element type; shell (bi-dimensional element), based on recommendations from the literature.

For some elements in the network, border conditions can be set (to define the retention of the blank), in this case we have chosen to carry out the detention as is the case of a retention element. Geometrically defined contact bodies are then attached to kinematic and contact characteristics. It further specifies the temporal evolution of the processing process by defining the increment of time and the number of increments required. Due to the specificity of the analyzed process, special attention is paid to the dimensional correlation of the geometry data of the finite element network with the rigid body kinematic and the time increment, in order to minimize the number of iterations of an increment due to the penetration of some knots beyond the boundary of the band contact. After repeated analysis, in the case presented in the present paper, it was practically eliminated the occurrence of such costly iterations (in computation time). Such optimization of the analysis is of course desirable in this preprocessing phase. A final stage of the preprocessing phase is the definition of the analysis options: the type of incremental equilibrium equation, the method of solving them, the solution convergence test, its tolerance, the initial loads, the type of contact, the width and position of the contact strip and the data the program writes in the output file. After entering all the data, write the input file for analysis.

The analysis phase is performed by the Ls-Dyna main module. After allocating the required space and data control, the program starts the increment cycle based on the time increment, with a first null increment for establishing the initial contact conditions and calculating the node load matrix. Load increments have the structure based on the sequence of two cycles, one related to the description of the plastic behavior of the blank material, and the other related to the iterative process of solving the system of equilibrium equations. Both cycles are broadly described above. Once the solution convergence condition is satisfied, the calculated data is written to the output file and the new contact conditions are analyzed based on the calculated nodal displacements. After imposing the displacements determined by the new tool-to-blank contact state, the calculation of the next time increment is made. The analysis is completed at the time of the complete deformation of the blank.

A final phase is postprocessing, in which the data written in the output file is processed in a graphical form to be interpreted. This phase of the post-processor Ls-Post is, in this case, extremely important due to the complex geometry of the processing system and the various display facilities that the program provides.

A processing system consisting of a $250 \times 250 \mathrm{~mm}$ rectangular blank, initially asymmetric stressed with a hemispherical die acting perpendicularly to its plane. The model of the processing system is shown in Figure 3. The blank is placed on the active plate and is secured by the blank holder by means of a $20 \mathrm{kN}$ retaining force applied uniformly over its 
surface. Thus, it is ensured the impossibility of sliding the blank through the clearance between the ring and the active plate, the process being analyzed being a process in which a certain depth of the piece is obtained by thinning the material.

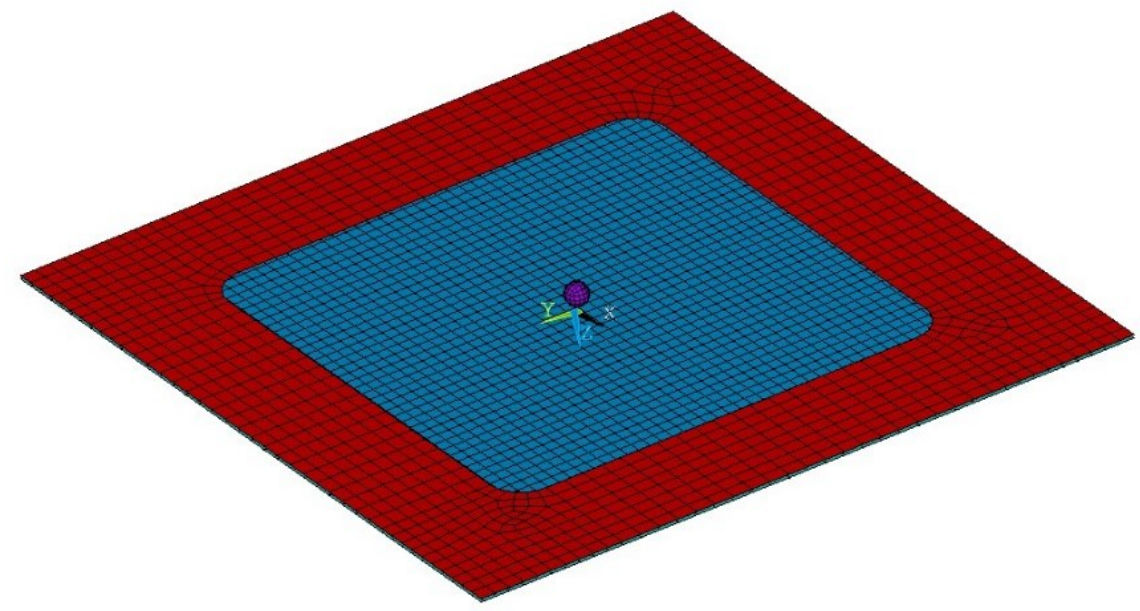

Fig. 3. The model of the processing system

It can, therefore, be seen that the finite element network associated with the geometry of the blank has a remeshing during simulation due to its exaggerated distortions so as to allow the analysis to proceed in good condition. The Ls-Dyna program provides the user with two indicators on which to meshing the blank: changing the angle between two adjacent finite elements or thinning the blank. We chose the second indicator because of the problem specific. As a rediscover level, we have chosen the third level to divide each finite element into a maximum of 64 elements until the end of the analysis, increasing the accuracy of the results, while maintaining an acceptable number of finite network elements to avoid overly long analysis times.

The blank, meshed as a solid deformable body, is made up of thin membrane elements (Thin Shell 163). The associated element type is described [8] as a four-node element having 12 degrees of freedom in each node and which can only be used in explicit analyses such as this one. The degrees of freedom associated with each node are represented by: nodal displacements, global rotation, velocities and nodal accelerations. Bilinear interpolation is used for coordinates, displacements and rotation. Specific deformations are calculated from the displacement field and the curve in the spin field. Specific transverse deformations are calculated at the middle of the sides and are interpolated at the integration points. In the analysis presented here, 9 integration points were used on the element thickness.

After the meshing of all the bodies forming the deformation system, four parts were made which defined the active plate, the die, the retaining ring and the blank. One part is the generic name used to define the set of geometry, the finite element network, the real constants and the materials for each of the elements of the deformation system. For the part that defines the active plate, all degrees of freedom were canceled: three translations and three rotation, for the one that defines the die, all rotations and translations of the $\mathrm{O}_{\mathrm{x}}$ and $\mathrm{O}_{\mathrm{y}}$ directions (both in the initial plane of the board) were canceled, leaving only the translation in the $\mathrm{O}_{z}$ direction (perpendicular to the plane of the board). The part associated with the retaining ring has exactly the same degrees of freedom as the die and can only make the translation in the direction of $\mathrm{O}_{\mathrm{z}}$. For all parts that define rigid bodies it was necessary to 
enter the data that define only their elastic behavior, namely the longitudinal elastic modulus, $\mathrm{E}=210 \mathrm{GPa}$ and the cross-contraction coefficient, $v=0.3$.

The type of material associated with the blank elements is MAT_89 (Material Plasticity Polymer). This type of material is an elasto-plastic material where true stress and arbitrary strain rate dependency curves can be defined. Since the process is slow and the deformation rate is reduced, the strain rate dependency curve is not introduced. For the true stress - true strain curves, unlike other materials (e.g. steels), the plastic component of the deformation is not reduced to this type of material. The elastic modulus considered is $\mathrm{E}_{\mathrm{PA}}=2.5 \mathrm{GPa}$, respectively $\mathrm{E}_{\mathrm{HDPE}}=0.8 \mathrm{GPa}$, and Poisson coefficient, $\mathrm{V}_{\mathrm{PA}}=0.39$ for polyamide and $\mathrm{V}_{\mathrm{PA}}=$ 0.46 for high density polyethylene. Introduced density of polyamide was $\rho_{\mathrm{PA}}=1.14 \mathrm{e}^{-9}$ tone/ $\mathrm{mm}^{3}$ and of polyethylene $\rho_{\mathrm{HDPE}}=0.95 \mathrm{e}^{-9}$ tone $/ \mathrm{mm} 3$. The criterion used to the occurrence of rupture was that the maximum effective plastic deformation. Thus, the $\varepsilon_{\mathrm{PA}, \max }=1.25 \mathrm{~mm} /$ $\mathrm{mm}$ for polyamide and $\varepsilon_{\mathrm{HPE}, \max }=5.02 \mathrm{~mm} / \mathrm{mm}$ values were introduced.

The physical process was performed with lubrication, the contact was idealized by using a static friction coefficient, $\mu=0.1$, which is also intended for simulation of the Erichsen, Olsen deformability tests, to which the present analysis has many similarities. The time increment used is $4 \mathrm{e}^{-07}$ seconds. The contact tolerance is $0.01 \mathrm{~mm}$ and is distributed equally around the outer surface of the blank. The force of detachment of the knots in contact is considered $1 \mathrm{e}^{-12} \mathrm{kN}$, so practically the smallest pulling force leads to breaking the contact. This is necessary because of the very frequent contact changes that occur in the type of this type of analysis.

For the most part, the efficiency of calculating Belytschko-Lin-Tsay elements (used in the present analysis) and Hughes-Liu subintegrates derives from the fact that they use the quadrature at a point in the plane of the element. This leads to deformations of the finite elements, deformations giving the quadrilateral finite element a similar appearance to a hourglass. This is unfavorable and needs to be removed. For this, the Ls-Dyna program uses Hourglass energy control. This energy must be limited to a maximum of $10 \%$ of the total energy consumed in the process.

\section{Results of the FEM analysis}

The processing system model, as defined above, was run on a 8-core graphics station and a total of $12 \mathrm{~Gb}$ of RAM. Running the program for the two variants of materials required considerable time. Thus, to complete the computation increments it took between 84 and 86 hours. This is fully justified by the magnitude of the problem by considering all three types of nonlinearities and the large number of time increments required to simulate the relative tool-blank movement.

The results of the calculation were written in the output file at every 100 increments for strain, stress and relative thinning, respectively for each 1000 increments for "history" results (forces, energies, etc.). The graphical data interpretation was performed with the LsPost postprocessor.

The results of the FEM analyses for the two types of studied materials are summarized in Table 2, under a number of quantitative aspects. Thus, the following characteristic sizes were chosen for study: maximum major strain, maximum minor strain, maximum thickness reduction, forces in the three directions and external work.

The state of strains and deformations obtained by numerical simulation indicates the possibility of reaching the plastic state of stress in blank (according to the Von Misses plasticity criterion) on its entire surface, but the pronounced localization of the stress in the die area is emphasized. This leads to the local appearance of higher tensions and deformations. 
Table 1. Results of the uniaxial tensile tests

\begin{tabular}{|c|c|c|c|}
\hline & $\begin{array}{c}\text { Longitudinal } \\
\text { modulus [MPa] }\end{array}$ & $\begin{array}{c}\text { Maximum } \\
\text { engineering } \\
\text { stress [MPa] }\end{array}$ & $\begin{array}{c}\text { Maximum engineering } \\
\text { strain [\%] }\end{array}$ \\
\hline Polyamide & 2505 & 41.04 & 135.29 \\
\hline Polyethylene & 803 & 24.73 & 502.49 \\
\hline
\end{tabular}

Table 2. Results of the numerical analysis

\begin{tabular}{|c|c|c|c|c|c|c|c|}
\hline & $\begin{array}{c}\text { Thickness } \\
\text { reduction } \\
{[\%]}\end{array}$ & $\begin{array}{c}\text { Major } \\
\text { strain } \\
{[\mathrm{mm} / \mathrm{mm}]}\end{array}$ & $\begin{array}{c}\text { Minor } \\
\text { strain } \\
{[\mathrm{mm} / \mathrm{mm}]}\end{array}$ & Fz [N] & Fx [N] & Fy [N] & L [kJ] \\
\hline Polyamide & 42.72 & 0.6174 & 0.2277 & 269.1 & 71.068 & 94.102 & 189.84 \\
\hline Polyethylene & 42.00 & 0.6429 & 0.2154 & 125.35 & 28.927 & 42.91 & 62.85 \\
\hline
\end{tabular}

\section{Conclusions}

From the analysis of the images 4 to 9 (the major strain, minor strain and thickness reduction maps) for the two materials it is observed that the strains and the thickness reduction maps show a higher degree of uniformity on the polyethylene parts walls compared to the polyamides due to the elongation higher than that of polyamides. It is noted that the maximum values of major strain, minor strain and thickness reduction are close for the two cases with a slight increase for polyethylene. In the major strain case, if major strain and thickness reduction have maximum values on the lateral wall area, it is noticed that maximum values for minor strain occur in the corners of the specimen.

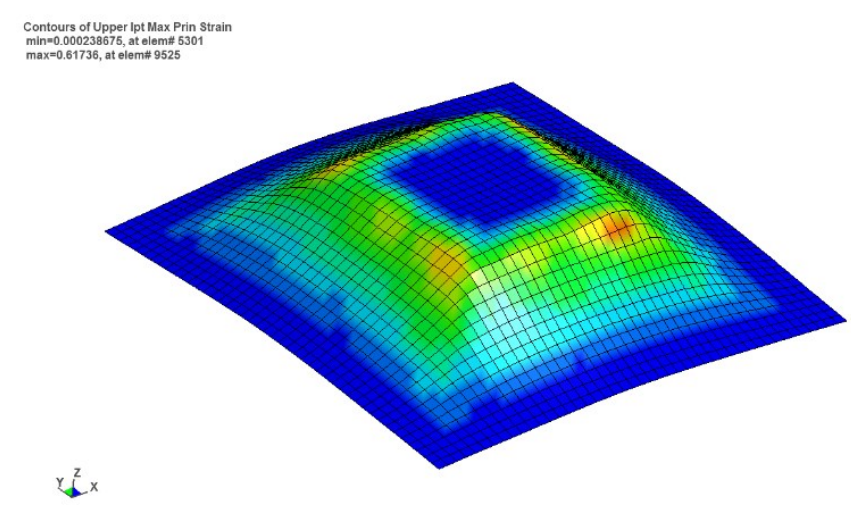

Fig. 4. Major strain map for polyamide 


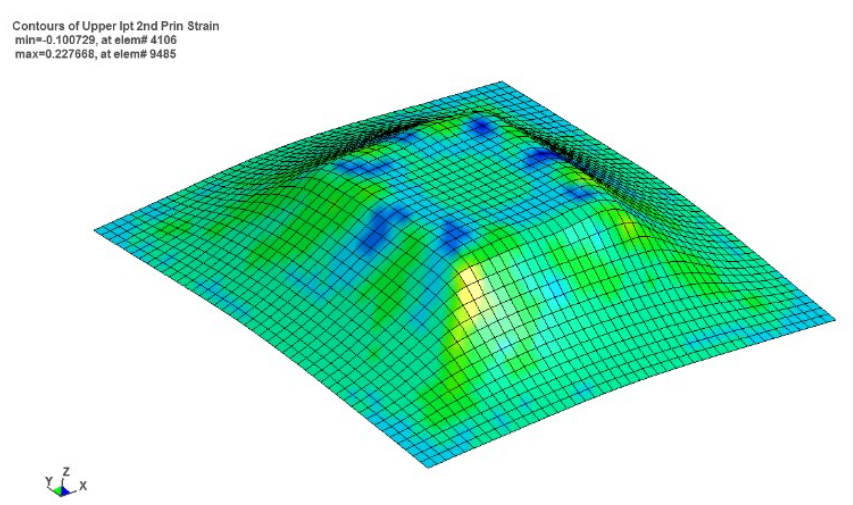

Fig. 5. Minor strain map for polyamide

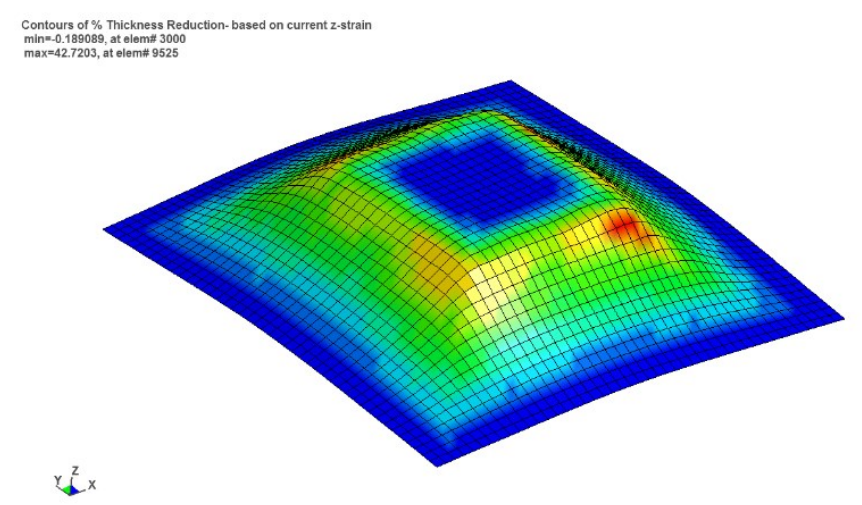

Fringe Levels

$4.272 e+01$

$3.843 \mathrm{e}+01]$

$2.985 \mathrm{e}+01$

2. $2.1266 \mathrm{e}+01$

$1.697 \mathrm{e}+01$ ]

$1.268 \mathrm{e}+01$

$8.393 e+00$

$4.102 \mathrm{e}+00$
$-1.891 \mathrm{e}-01$

Fig. 6. Thickness reduction for polyamide

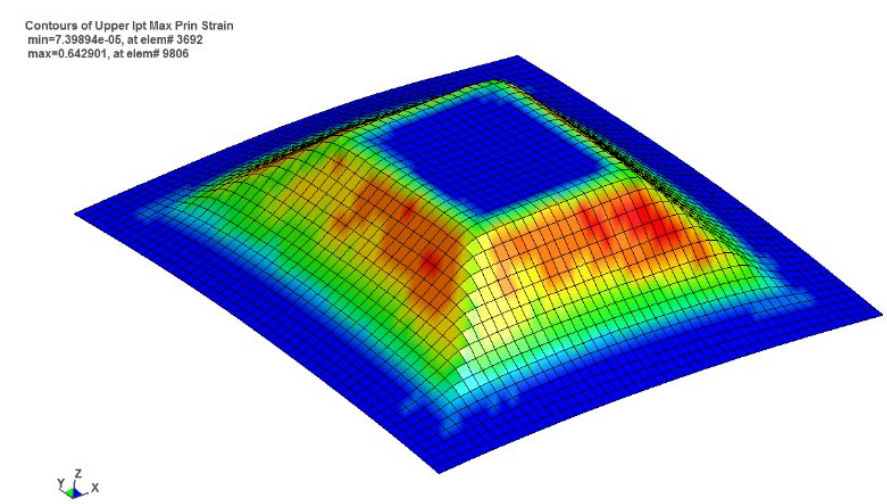

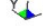

Fig. 7. Major strain for polyethylene 

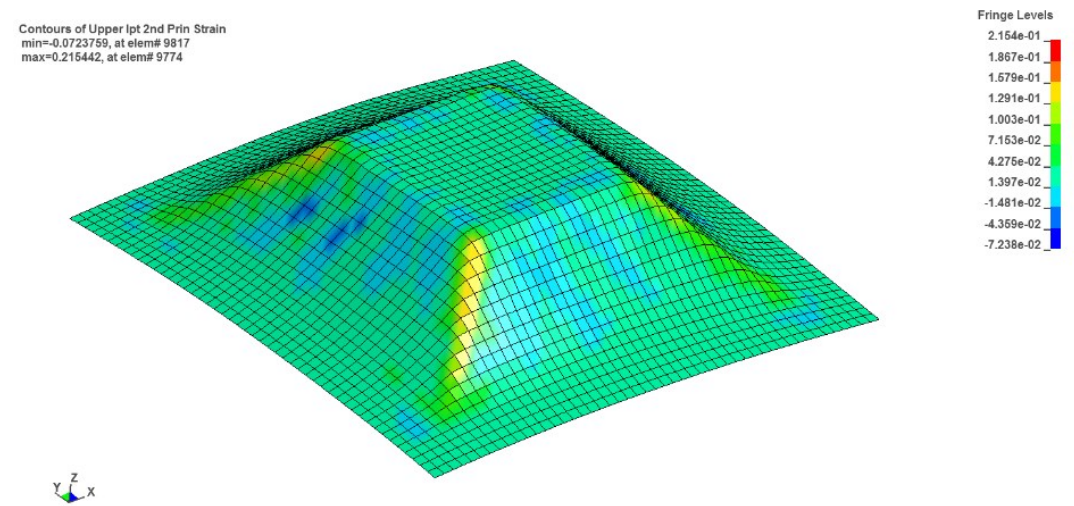

Fig. 8. Minor strain for polyethylene

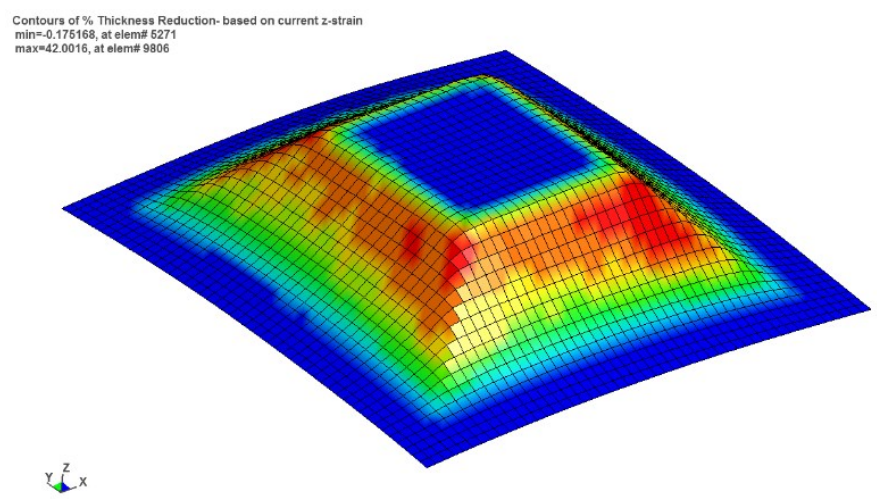

$x^{2}$

Fig. 9. Thickness reduction for polyethylene

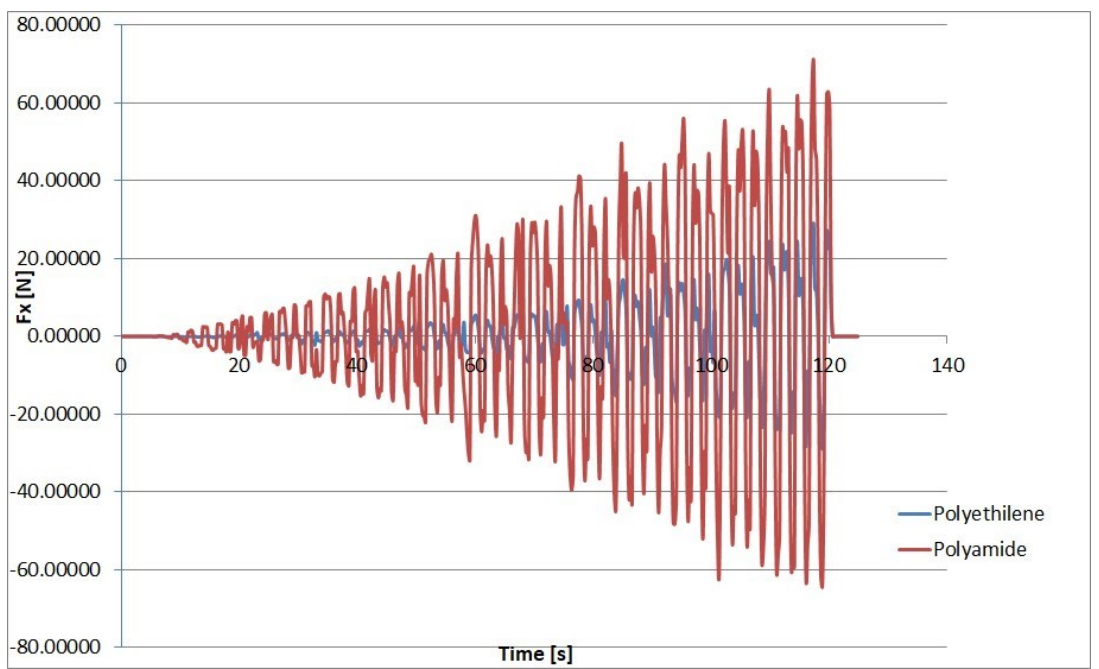

Fig. 10. Forces in horizontal direction 


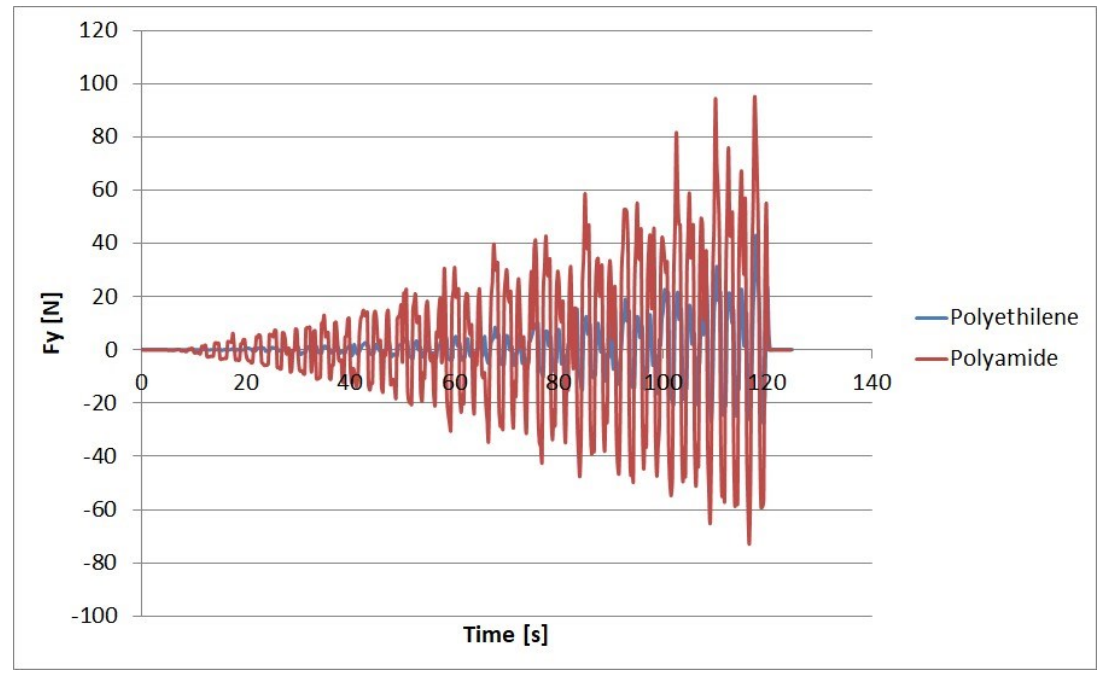

Fig. 11. Forces in vertical direction

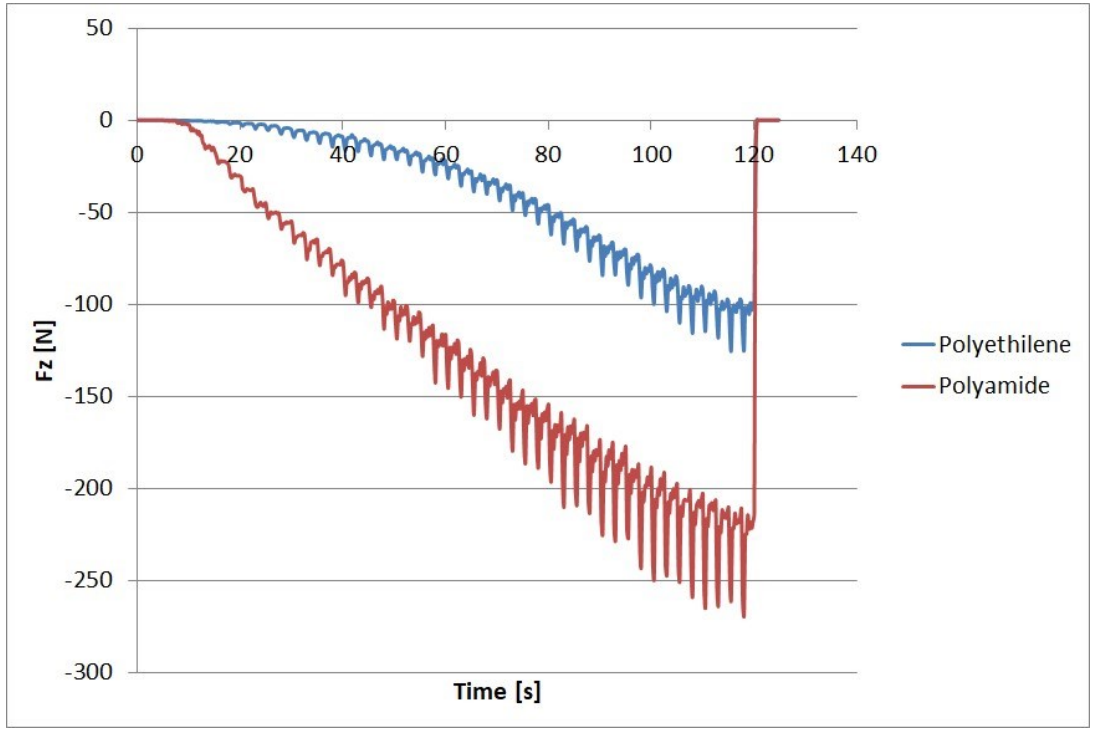

Fig. 12. Forces in Z-direction

Regarding the energy of the process, it is observed that the maximum value of the forces in both vertical and horizontal direction is more than double in the case of polyamides than polyethylene (figures 10 to 12 ).

The same conclusion can be drawn in the case of external work whose value is 3 times higher for polyamides than for polyethylene (fig.13). 


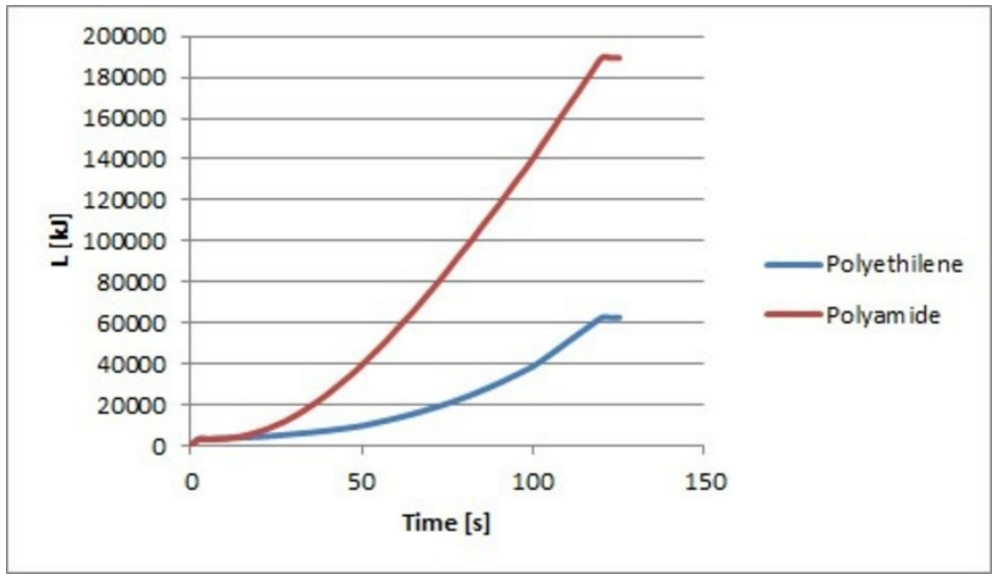

Fig. 13. External work

\section{References}

1. A.K. Behera, R.A. De Sousa, G. Ingarao, V. Oleksik, J Manuf Process, 27, 37 (2017)

2. V. Oleksik, The 11th International Conference on Technology of Plasticity - 2014, Nagoya, Japan, October 19-24, Procedia Engi, 81, 2280 (2014)

3. D. Fritzen, A. Daleffe, G. Do Santos De Lucca, J. Castelan, L. Schaeffer, R.J. Alves De Sousa. Int J Mater For, 11(3), 389 (2018)

4. D. Nasulea, G. Oancea, Stroj Vestn-J Mech E, 64(10), 643 (2018)

5. S.G. Racz, R.E. Breaz, M. Tera, C. Girjob, C. Biris, A.L. Chicea, O. Bologa , Metals, 8(8), 30 (2018)

6. I. Bagudanch, M.L. Garcia-Romeu, G. Centeno, A. Elías-Zúñiga, J. Ciuarana. J Mater Process Technol, 219, 221 (2015)

7. I. Bagudanch, M. L. Garcia-Romeu, I. Ferrer, J. Ciurana, Rapid Prototyp J, 24(1), 120 (2018)

8. $* * *$ LS-DYNA v 11. Keyword user's manual. Livermore Software Technology Corporation, 1, 2, 3 (2018) 